aber durchaus ironiebewussten Schreibstil ist hier eine hervorragende Beschreibung der FDP gelungen. Der Mehrwert bei Dittberner liegt demgegenüber in der kurzen und gefärbten Darstellung der Geschichte der Freidemokraten. Für den kritischen Leser ergibt sich hieraus eine Innenansicht, die durchaus eine Bereicherung sein kann. Beide Bände stehen mit den genannten Abstrichen einer breiten Leserschaft offen - egal ob etablierter Parteienforscher, Lehrender oder Student.

Christian Nestler

\title{
Frauen in der CDU: reiche Erfahrungsberichte
}

Neuss, Beate und Hildigund Neubert (Hrsg.): Mut zur Verantwortung. Frauen gestalten die Politik der CDU, Böhlau Verlag, Köln / Weimar / Wien 2013, 717 Seiten, € 39,90.

Der von Beate Neuss und Hildigund Neubert herausgegebene 700 Seiten starke Band ist ein spannendes Lesebuch für alle, die sich für die Menschen in der Politik interessieren. 58 prominente Frauen aus der CDU, nach Geburtsjahren geordnet, geben in eigenen Texten oder in autorisierten Interviews Auskunft über ihre politische Sozialisation. Sie berichten, warum sie sich für die CDU entschieden haben, wie sie von der Partei und ihren Vereinigungen aufgenommen wurden und was sie durch ihren Einsatz auf der kommunalpolitischen Ebene gelernt haben. Gefragt wurde, wann sie angefangen haben, sich mit der Interessenvertretung von Frauen auseinanderzusetzen, was sie von Quoten halten und was die Frauen Union für sie bedeutet. Sie geben Auskunft zu Politikinhalten, ihrem Politikstil, zur Vereinbarkeit von Familie, Beruf und Politik und schließen mit Empfehlungen an junge Frauen.

Die eindringlichen, durchdachten eigenen Texte und Interviews überraschen in ihrer Vielfalt, denn so verschieden wie die Frauen sind auch ihre Wege. Es gibt Frauen:

- die die Chance von Vakanzen nutzten, gesucht, geworben, gefragt und gefördert wurden,

- die gestalten wollten und sich Schritt für Schritt auf der „Ochsentour“ von der Elternvertretung über das kommunale Mandat in den Landtag, den Bundestag und/oder das Europäische Parlament hocharbeiteten und jede Position verteidigen mussten und jene, - die als Seiteneinsteigerinnen den „Parteiarbeiterinnen“ vorgezogen wurden.

Noch in der Weimarer Republik sozialisierte Einzelkämpferinnen, studierte Proporzhausfrauen der 1920er Jahrgänge, privilegierte Seiteneinsteigerinnen und junge Politikerinnen informieren über den selten thematisierten Alltag der daueraktiven Funktions- und Mandatsträger, der „2. Klasse“ (Wilhelm Hennis). Dies gilt besonders für die Landtags- und Bundestagsabgeordneten (Renate Hellwig, Ilse Falck, Eva Wybrands und Eva Möllring), aber auch für Dorothee Wilms, die „aus einer anderen Zeit“ über die Ochsentour berichtet. Die Spitzenpolitikerinnen äußern sich verhalten kontrolliert in Interviews.

Die Texte spiegeln die unterschiedlichen Bedingungen der Kriegsgeneration, der mittleren Generation der Kriegs- und Nachkriegskinder, der 1950er Jahrgänge, die den Essener Beschlüssen folgten. Wie sehr sich die Situation für Frauen verändert hat, nachdem der Vorsitzende Helmut Kohl 1985 Rita Süssmuth zur Ministerin für Jugend, Familie, Frauen und Gesundheit berief, und sie den Vorsitz der Frauen Union übernahm, zeigt Ingrid Sehrbrock, die von 1989 bis 1997 Bundesgeschäftsführerin der Frauen Union war. Die nach 1960 geborenen Frauen finden Vorbilder, Angebote zur Zusammenarbeit und Organisation 
nicht nur in der Frauen Union unter Rita Süssmuth und Maria Böhmer, sondern auch in der Gruppe der Frauen im Bundestag, die mit ihren Sprecherinnen im Fraktionsvorstand vertreten ist und in direktem Kontakt mit der Bundeskanzlerin Angela Merkel steht.

Die Entscheidung für das Quorum 1996 wird rückblickend auch von früheren Gegnerinnen begrüßt, da sie Frauen die Chance gegeben habe, sich mit ihren Themen einzubringen. Sie folgt nach langem Kampf den Erfahrungen des Landesverbandes Rheinpfalz. Ingrid Sehrbrock berichtet, wie Helmut Kohl im Bundesvorstand „die Konsequenzen der Drittelquote erläuterte. Auf die Frage eines Vorstandsmitglieds, ob allen Ernstes neben den Listen auch Wahlkreise quotiert werden sollten, erklärte er sinngemäß: In Rheinland-Pfalz haben wir immer darauf geachtet, dass wir Landwirte, Winzer und Protestanten in ausreichender Zahl als Kandidaten hatten. Und wenn wir keine hatten, haben wir welche gesucht. Und das machen wir jetzt auch für Frauen“"(S. 421). Die Wirkung dieser Haltung ist an den Berichten von Roswitha Verhülsdonk, Maria Herr-Beck, Maria Böhmer, Susanne Tiemann und Julia Klöckner abzulesen. Sie hat die CDU so verändert, dass die Partei „mit all ihren Personen im Alltag viel moderner ist als ihr Image“ (S. 687). Doch ohne Männer geht es nicht. Am erfolgreichsten waren im Doppelpack erarbeitete und vorgetragene Initiativen. Schwierig wird es, wenn sich die Männer der Partnerschaft verweigern. Wie viel Überzeugungsarbeit unter den Frauen selbst, aber vor allem in der männlich strukturierten Partei und Gesellschaft geleistet werden musste, wird daran deutlich, dass Kinderbetreuung für Abgeordnete oder gar Mütterzeiten für Ministerinnen nicht bedacht und nirgendwo vorgesehen waren (Katherina Reiche, Hildegard Müller, Kristina Schröder).

Die Beiträge von alleinstehenden, verheirateten, verwitweten kinderlosen Politikerinnen zeigen beispielhaft, wie die Herausforderung „die Vielfalt weiblicher Lebensmodelle zu akzeptieren, ohne diejenigen zu diskreditieren, die bisher das konservative Familienmodell gelebt hatten" (S. 421), von Frauen Union und CDU gemeistert wurde.

Die zeitgeschichtliche Einordnung und Ost-West-Unterschiede thematisieren Else Ackermann - am Kampf zum Rentenüberleitungsgesetz und zur Restitution - und Sabine Bergmann-Pohl mit Stasi-Erfahrung in den eigenen Reihen. Deutlich wirken ihr Selbstbewusstsein durch Berufstätigkeit, aber auch die Erfahrung als Außenseiter und die Suche nach Nischen auf das Verhalten der Frauen aus den neuen Bundesländern. Es fällt auf, wie viele der Frauen aus Rheinland-Pfalz, Nordrhein-Westfalen, Hessen und Niedersachsen kommen, während Bremen und Mecklenburg-Vorpommern gar nicht vertreten sind. Frauen haben es weiter schwer in Landesverbänden, die keine Listenplätze zu vergeben haben, denn gerade im ehrenamtlichen Teil der Ochsentour sind Familie, Beruf und Politik nur mit - immer noch seltener - Unterstützung des Ehemanns (Erika Steinbach, Annegret Kramp-Karrenbauer, Christine Lieberknecht) zu vereinen. Die Frauen beschreiben ihren Politikstil als sachorientiert und effizient. Selbstdarstellung und Ritualen geben sie selten Raum. Die jüngeren gut ausgebildeten Frauen empfehlen, den eigenen Weg zu suchen und selbstbewusst zu gehen, Chancen aus der Konjunktur der „weichen Themen“ zu nutzen und dabei das Licht nicht unter den Scheffel zu stellen. Da man politische Karrieren nicht planen kann, sei ein festes berufliches Standbein nötig (Godelieve Quisthoudt-Rowohl, S. 413). In den Berichten überwiegt die positive Lebensbilanz. Das macht Mut, sich in Gesellschaft und Politik einzubringen.

Es bleibt allerdings den Lesern als lockende Aufgabe überlassen, die vielfältigen Informationen selbst auszuwerten. 